\title{
Effects of sex steroids on cardiovascular risk profile in female to male transsexuals with cross-sex hormone treatment.
}

Gloria Aranda 1,2, Mireia Mora 1,2, Felicia A. Hanzu 1,2, Josep Vera 3, Emilio Ortega ${ }^{1}$, Irene Halperin ${ }^{1,2}$

1. Endocrinology Department, HCB/IDIBAPS/CIBERDEM, Barcelona, Spain. ${ }^{2}$ Endocrine Disorder Laboratory IDIBAPS/CIBERDEM, Barcelona, Spain. ${ }^{3}$ IDIBAPS/CIBERDEM, Barcelona, Spain.

\section{Background}

In transsexualism, cross-sex hormone treatment (CHT) both improves and impairs several surrogate cardiovascular risk markers in female to male (FtoM) GID. Few randomized trials on $\mathrm{CHT}$ with long follow-up and control group are available, and present evidence is inconsistent

\section{Aim}

To assess changes in metabolic and cardiovascular risk after 12 months of CHT compared with biological sex in FtoM GID

\section{Methods}

Prospective observational study, including 20 FtoM GID, attended in the Gender Identity Unit (GIU) of Hospital Clinic from July 2012 to November 2013.

CHT in FtoM consisted of intramuscular testosterone undecanoate $(1000 \mathrm{mg}$ every 2 3 months), except for one who received transdermal testosterone $(50 \mathrm{mg} /$ day).

Contraindications for CHT were ruled out; subjects had not previously received hormonal treatment, and had no history of cardiovascular disease or HIV.

Anthropometry and body composition by DEXA, hormonal, metabolic and coagulation parameters were assessed at baseline, and 6 and 12 months of CHT. Endothelial dysfunction was evaluated by flow-mediated dilation (FMD) and Intima-media thickness, (IMT) by carotid ultrasound at baseline, 6 and 12 months.

\section{Results}

\section{Changes after 6 and 12 months of CHT}

Anthropometry and body composition: increased BMI $(p=0.001)$, decreased total fat mass $(p=0.050)$, increased total lean mass $(p=0.007)$ and decreased ginecoid fat distribution $(p=0.008)$.

Hormonal: decreased LH $(p=0.008)$, SHBG $(p<0.001)$ and increased testosterone $(p<0.001)$.

Metabolism: increased total cholesterol $(p=0.043)$, LDL-c $(p=0.019)$ and triglycerides $(p=0.001)$, and decreased HDL $(p=0.035)$; increased homocysteine $(p=0.003)$ and Leucocytes $(p<0.001)$.

Coagulation: increased hemoglobin $(p<0.001)$ and hematocrit $(p<0.001)$ and decreased platelets $(p=0.011)$.

No changes in FMD or IMT were observed.

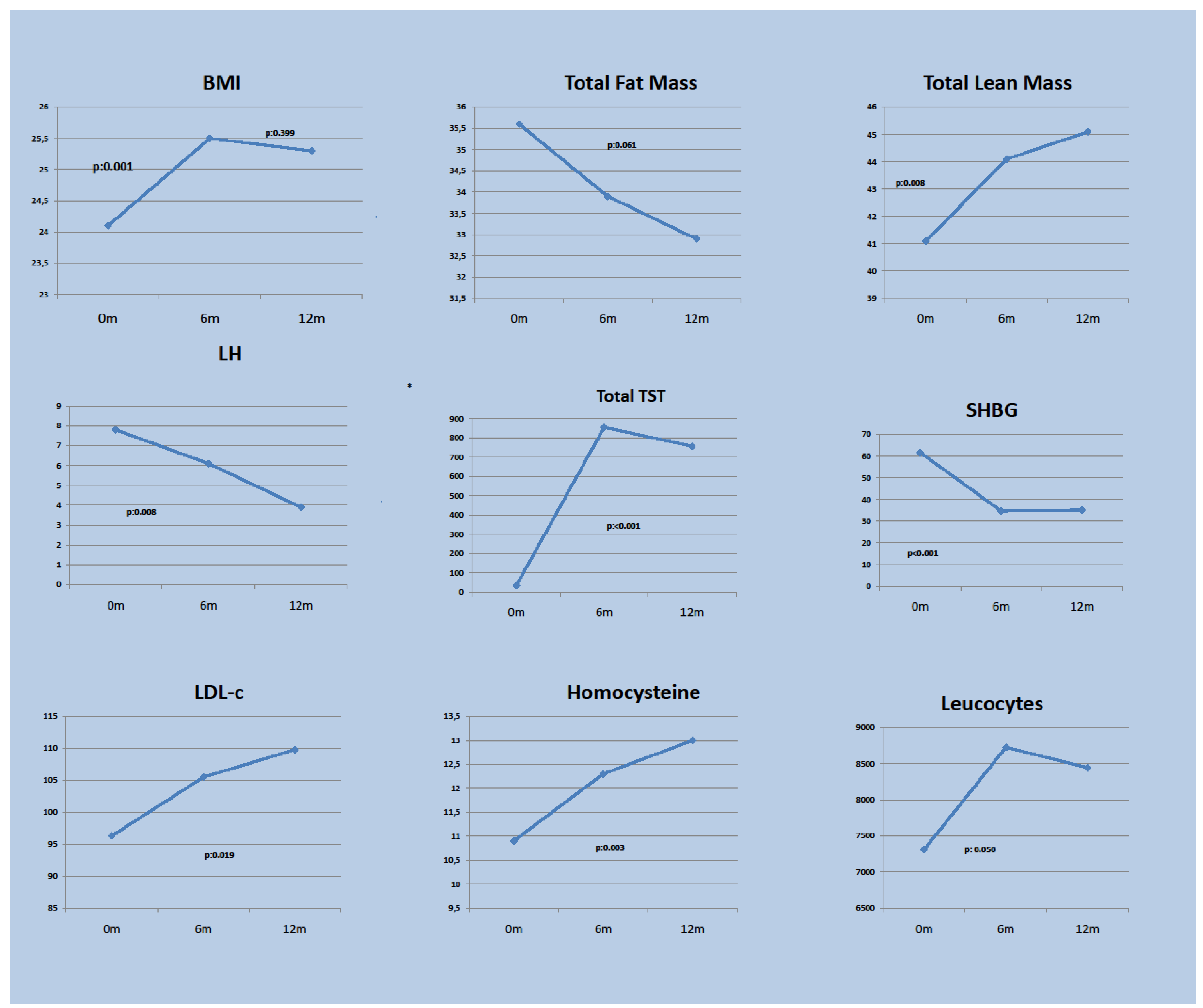

\section{Conclusions}

CHT in FtoM GID has negative effects in metabolic profile in the first 12 months of treatment which could be associated to an increase of cardiovascular risk. 